\title{
IgA nephropathy presenting as rapidly progressive glomerulonephritis following first dose of COVID-19 vaccine
}

\author{
Olivier Niel ${ }^{1,2}\left(\right.$ Cosmin Florescu $^{3}$
}

Received: 15 October 2021 / Revised: 24 October 2021 / Accepted: 25 October 2021 / Published online: 16 November 2021

(c) The Author(s), under exclusive licence to International Pediatric Nephrology Association 2021

To the Editors,

Coronavirus disease 2019 (COVID-19) has recently been associated with kidney disease in pediatric patients [1]. Interestingly, COVID-19 vaccination has also been described as a potential trigger of acute kidney injury (AKI); in particular, IgA nephropathy (IgAN) flare-ups following mRNA COVID-19 vaccination have been reported in adult [2] and pediatric patients [3]. However, the pediatric cases published so far described patients suffering from mild IgAN flare-ups occurring after the second dose of the COVID-19 vaccine.

Here, we describe the development of de novo IgAN occurring within $24 \mathrm{~h}$ following the first dose of mRNA COVID-19 vaccine. The patient was 13 years old, with no relevant medical history. In particular, she had no history of COVID-19 infection (SARS-CoV-2 PCR and serology were negative), and she never had reacted to any previous vaccinations. Within $24 \mathrm{~h}$ following the first dose of the Pfizer mRNA COVID-19 vaccine, she developed fever, asthenia, and muscle pain. Macroscopic hematuria was also reported. At admission, clinical examination showed a mild streptococcus-negative pharyngitis. Serum creatinine was $3.57 \mathrm{mg} / \mathrm{dl}$, and blood urea nitrogen was $96 \mathrm{mg} / \mathrm{dl}$. Macroscopic hematuria was accompanied by nephrotic range proteinuria $(3.88 \mathrm{~g} / \mathrm{l})$. Other etiologic investigations came back negative, including immunological and infectious testing.

Kidney biopsy performed at day 4 post-vaccination is shown in Fig. 1. The estimated Oxford score was

Olivier Niel

niel.olivier@chl.lu

1 Pediatric Nephrology, Centre Hospitalier de Luxembourg, 5 rue Barblé, L1210 Luxembourg, Luxembourg

2 Faculty of Science, Technology and Medicine, University of Luxembourg, 2 Avenue de l'Université, L4365 Esch-sur-Alzette, Luxembourg

3 National Center of Pathology, Laboratoire National de Santé, 1 rue Rech, L3555 Dudelange, Luxembourg
M1E1S0T0. Kidney function rapidly deteriorated, and the patient became oliguric; as a consequence, hemodialysis was started. Treatment consisted of 3 IV methylprednisolone pulses, followed by oral prednisone. Kidney function improved progressively; hemodialysis was stopped at 5 days post-vaccination. At 11 days post-vaccination, serum creatinine was down to $1.9 \mathrm{mg} / \mathrm{dl}$. At 30 days post-vaccination, serum creatinine had returned to almost normal values $(0.86 \mathrm{mg} / \mathrm{dl}$, leading to a GFR according to the Schwartz formula of $82 \mathrm{ml} / \mathrm{min}$ ). Microscopic hematuria and a slight proteinuria persisted.

Unlike previous observations [2, 3], this report suggests that development of IgAN following COVID-19 vaccination may occur after the first dose of vaccine, and may present as a rapidly progressive glomerulonephritis leading to severe AKI. As with other case reports, COVID-19 vaccine responsibility in the IgAN flare-up reported here remains difficult to establish. Therefore, it appears essential to precisely assess the risk-benefit ratio of COVID-19 vaccination in all pediatric age groups, and to carefully plan COVID-19 vaccination in patients with chronic kidney disease at risk of relapsing.

Acknowledgements The authors would like to thank Dr. Benchalel, Dr. Olivier, and Dr. De La Fuente for their help in the management of the patient.

Author contribution All authors contributed to drafting, reviewing, and revising this paper. 

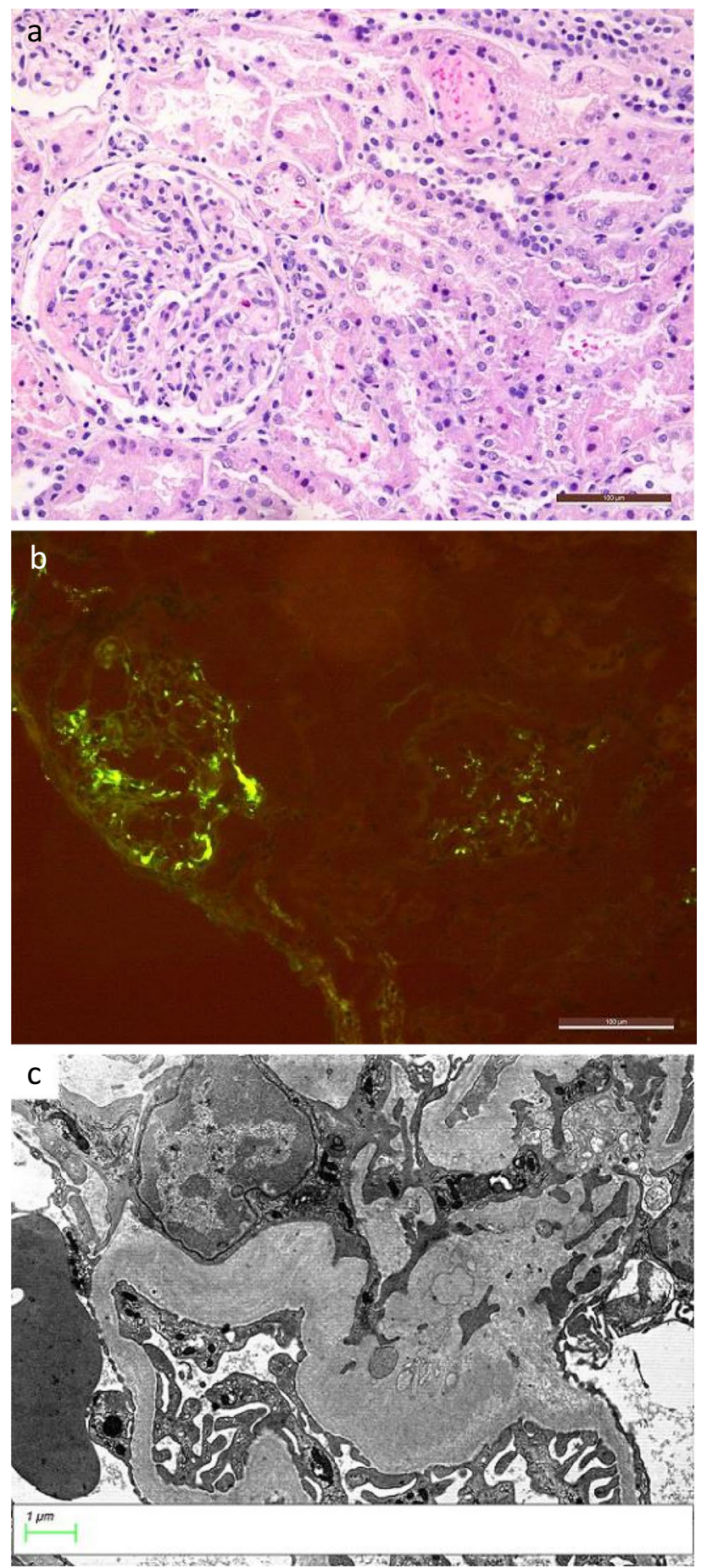

Fig. 1 Pathology showing IgA nephropathy, Oxford score M1E1S0T0. a Optical microscopy (scale bar $100 \mu \mathrm{m}$ ). Mesangial and endocapillary proliferation. No constituted crescents were observed, but fibrin deposits were present in the Bowman space of most of the observed glomeruli. No evidence of renal scarring from previous kidney injury could be detected. b Immunofluorescence (scale bar $100 \mu \mathrm{m})$. Diffuse mesangial IgA and C3 deposits. Absence of other deposits. c Electron microscopy (scale bar $1 \mu \mathrm{m}$ ). Diffuse mesangial deposits

\section{Declarations}

Informed consent Informed consent was obtained from legal guardians.

Conflict of interest The authors declare no competing interests.

\section{References}

1. Serafinelli J, Mastrangelo A, Morello W, Cerioni VF, Salim A, Nebuloni M, Montini G (2021) Kidney involvement and histological findings in two pediatric COVID-19 patients. Pediatr Nephrol 36:3789-3793

2. Rahim SEG, Lin JT, Wang J (2021) A case of gross hematuria and IgA nephropathy flare-up following SARS-CoV-2 vaccination. Kidney Int 100:238

3. Hanna C, Hernandez Herrera LP, Bu L, Kizilbash S, Najera L, Rheault MN, Czyzyk J, Kouri AM (2021) IgA nephropathy presenting as macroscopic hematuria in 2 pediatric patients after receiving the Pfizer COVID-19 vaccine. Kidney Int 100:705-706. https://doi.org/10.1016/j.kint.2021.06.032

Publisher's note Springer Nature remains neutral with regard to jurisdictional claims in published maps and institutional affiliations. 